\title{
Orbital Complication of Allergic Fungal Rhino-sinusitis
}

\section{Ali H. Alzarei* and Kholood S. Assiri}

The Departments of Otorhinolaryngology, Head \& Neck Surgery, Aseer Central Hospital, Abha City, Saudi Arabia

\begin{abstract}
Background: Allergic fungal rhino-sinusitis(AFRS) is a benign noninvasive sinus disease related to hypersensitivity to fungal inhalation occurs in young immunocompetent individuals. AFRS can present with serious orbital complication.

Aim: This study was conducted To determine the common features of orbital complications due to allergic fungal rhino-sinusitis .

Methods: A retrospective study of 60 patients. At Aseer central Hospital ( Abha, Saudi Arabia) from 2010 to 2013. All diagnosed and treated for allergic fungal rhino-sinusitis. Diagnosis was made based on Bent - Kuhn criteria and analysis of CT scan reports of these patients, the data coded-edited-analyzed using SPSS IBM version 22.

Results: Out of 60 patients 27 had orbital complications, Eye proptosis $63 \%$, diplopia $22 \%$ and Unilateral complete blindness $15 \%$.

Fungal hyphae was not demonstrated histopathologically in any of these patients. The presence of fungal mucin was prevalent in all these patients.

Eye proptosis has good dramatic respond to surgical and post operative treatment while diplopia take longer time to improve after surgical and medical treatment.

Conclusion: Allergic fungal rhino-sinusitis (AFRS) can present with serious orbital complication: eye proptosis, diplopia, and unilateral complete blindness. All patients with orbital involvement should be evaluated clinically and radiologically for sinus disease even in the absent of stigma of rhino-sinusitis. Early diagnoses and immediate treatment are extremely essential to prevent serious complication such as visual loss.
\end{abstract}

Publication History: Received: March 14, 2016 Accepted: April 01, 2016 Published: April 04, 2016

\section{Keywords:}

Allergic fungal, Rhino-sinusitis, Orbital complication

\section{Introduction}

Young et al in 1978 described allergic fungal sinusitis [1]. They described a case with pan sinusitis with bone erosion. They described the contents of maxillary sinuses to be abundant in mucous admixed with eosinophils, necrotic debris and scattered fractured fungal hyphae. The condition "Allergic fungal sinusitis" as a clinical entity was described in 1981 by Millar et al. [2]. Two years later, Katzenstein et al independently observed the pathophysiologic resemblance between allergic bronchopulmonary aspergillosis and 7 cases of chronic fungal sinusitis. The term allergic fungal sinusitis was coined in 1989 [3]. Since then, extensive work has been carried out to explain the disease as an immunologically mediated disorder (IgE mediated) rather than a precursor of invasive fungal disease [4-7] . Allergic fungal sinusitis is a noninvasive, but vigorous, inflammatory response to mold that occurs in immunocompetent patients with chronic sinusitis and nasal polyposis. It typically occurs in patients who have a history of atopic disease [8]. The paranasal sinuses of these patients are characteristically filled with eosinophil rich mucin. Thick fungal debris and mucin having carbohydrate-rich glycoprotein develop in sinus cavity [9] .This mucin is characteristically known as "Allergic mucin". Patients with allergic fungal sinusitis commonly suffer from asthma. Studies reveal Asthma associated with AFS is estimated to range from $20 \%$ to $40 \%$ [10]. This condition is caused by $\operatorname{IgE}$ driven eosinophilic inflammation within the paranasal sinuses. The cytokines released from this inflammation are known to cause the classic features seen in allergic fungal sinusitis.

Clinical findings in these patients include:

1. Signs of nasal mucosal inflammation

2. Nasal polyposis

3. Facial disfigurement

4. Orbital abnormalities in form of proptosis, epiphora and visual loss
The Reported ophthalmic manifestations of allergic fungal sinusitis include proptosis, diplopia, blepharoptosis, epiphora, opthalmoplegia, orbital abscesses and rarely visual loss $[11,12]$. Visual loss associated with allergic fungal sinusitis is an uncommon finding (1.46\% to $3.7 \%)$ [13]. The pathophysiology of visual loss in patients with allergic fungal sinusitis has been proposed to be either through direct or indirect optic nerve compression, or through an inflammatory process that results in optic neuritis [13-14].

Compressive visual loss usually is a result of an initial venous occlusion with consequent tissue edema and nerve compression.

Diagnostic criteria for allergic fungal sinusitis:

1. Gross production of eosnophilic mucin containing non invasive fungal hyphae

2. Nasal polyposis

3. Characteristic radiological findings

4. Immuno competence

5. Allergic mucin [4]

Radiological characteristics of allergic fungal sinusitis :[15]

1. Classically asymmetrical involvement of paranasal sinuses are seen in plain radiographs and CT imaging.

*Corresponding Author: Dr. Ali H. Alzarei, The Departments of Otorhinolaryngology, Head \& Neck Surgery, Aseer Central Hospital, Abha City, Saudi Arabia, E-mail: drzizous2000@yahoo.com

Citation: Alzarei AH, Assiri KS (2016) Orbital Complication of Allergic Funga Rhino-sinusitis. Int J Clin Case Stud 2: 111. doi: http://dx.doi.org/10.15344/24552356/2016/111

Copyright: (c) 2016 Alzarei et al. This is an open-access article distributed under the terms of the Creative Commons Attribution License, which permits unrestricted use, distribution, and reproduction in any medium, provided the original author and source are credited. 
2. Bone erosion with extension of the disease to adjacent areas seen due to pressure effect [16].

3. Sinus expansion was associated with the presence of bone erosion [17] .

4. Heterogenous areas of signal intensities in sinus cavities filled with allergic mucin is seen in CT imaging. This is due to accumulation of heavy metals like iron and manganese.

5. 'Double density'sign is usually caused by the dense inspissated eosinophil-rich extramucosal allergic mucin, It should be noted that double density sign might also be seen with other forms of fungal sinusitis $[15,18]$.

Histological features of allergic fungal sinusitis:

Histology of allergic fungal mucin reveals the characteristic branching non invasive fungal hyphae within sheets of eosinophils and Charcot - Layden crystals. Histopathologic diagnosis requires both hematoxulin and eosin ( $\mathrm{H} \& \mathrm{E}$ ) and fungal stain such as Gomori's methenamine silver stain [19].

Over the past 2 decades, allergic fungal sinusitis (AFS) has become increasingly defined. Historically mistaken for a paranasal sinus tumor.

\section{Aim}

This study was conducted To determine the common features of orbital complications due to allergic fungal rhino-sinusitis.

\section{Materials and Methods}

A retrospective study of 60 patients at Aseer central Hospital ( Abha, Saudi Arabia) from 2010 to 2013 was done. All patients are diagnosed and treated for allergic fungal rhino-sinusitis. Diagnosis was made based on Bent-Kuhn criteria and analysis of CT scan reports of these patients.

Diagnosis was made based on Bent-Kuhn criteria and analysis of CT scan reports of these patients [4].

Bent and Kuhn proposed 5 criteria for the diagnosis of allergic fungal sinusitis.

(1) type I hypersensitivity (atopy) diagnosed by history, positive skin test, or serology(2) nasal polyposis(3) characteristic CT scan findings

(4) Positive fungal smear (This feature was not seen in any of the patients in our study) and (5) allergic mucin.

Following parameters were taken up for study: Incidence of orbital manifestations, Symptomatology and Outcome of orbital complications.

\section{Clinical findings in these patients include}

Signs of nasal mucosal inflammation, Nasal polyposis, Facial disfigurement, Orbital abnormalities.

The data coded-edited-analyzed using SPSS IBM version 22.

\section{Results}

Allergic fungal rhino-sinusitis commonly present with unilateral pan-sinusitis that might associate with orbital complications. 27 patients out of 60 had orbital complications.

Common ophthalmic manifestations present in these patients include: Eye proptosis $63 \%$, diplopia $22 \%$ and Unilateral complete blindness $15 \%$.

Fungal hyphae was not demonstrated histopathologically in any of these patients. The presence of fungal mucin was prevalent in all these patients.

- Eye proptosis has good dramatic respond to surgical and post operative treatment. diplopia take longer time to improve after surgical and medical treatment.

- Patients with allergic fungal sinusitis are immunocompetent individuals [2].

- They are also fairly young when compared to patients with other forms of invasive fungal sinusitis.

- Opthalmic manifestations are fairly common in these patients. A high degree of suspicion is necessary for diagnosing this condition.

- This disease is due to immunological reaction of the nasal mucosa against fungal proteins [20] .

- The presence of orbital periosteum is a deterrent to spread of these lesions into the orbit.

- Since allergic fungal sinusitis can cause disastrous orbital complications ranging from ophthalmoplegia to total blindness it should be considered as an emergency.

- Endoscopic debridement should be performed in these patients.

- Patients with orbital involvement will always seek the help of ophthalmologist in the first place.

- Opthalmologists should be aware of this potential problem.

\section{Conclusion}

- $\quad$ Allergic fungal rhino-sinusitis(AFRS) can present with serious orbital complication: eye proptosis, diplopia, and unilateral complete blindness

- All patients with orbital involvement should be evaluated clinically and radiologically for sinus disease even in the absent of stigma of rhinosinusitis.

- Initial diagnosis of allergic fungal sinusitis requires suspicion on the part of the ophthalmologist. Proptosis is the most common ophthalmic sign [21].

- Surgical debridement and empiric systemic antifungal therapy, followed by serial endoscopic evaluations, are the mainstays of treatment.

\section{Conflict of Interests}

There are no relevant conflict of interests to disclose regarding the publication of this paper.

\section{References}

1. Young CN, Swart JG, Ackermann D, Davidge-Pitts K (1978) Nasal obstruction and bone erosion caused by Drechslera hawaiiensis. J Laryngol Otol 92: 137-143. 
Citation: Alzarei AH, Assiri KS (2016) Orbital Complication of Allergic Fungal Rhino-sinusitis. Int J Clin Case Stud 2: 111. doi: http://dx.doi.org/10.15344/24552356/2016/111

2. Millar JW, Johnston A, Lamb D (19981)Allergic aspergillosis of the maxillary sinuses. InThorax 36: 710-710.

3. Robson JM, Hogan PG, Benn RA, Gatenby PA (1989) Allergic fungal sinusitis presenting as a paranasal sinus tumour. Aust N Z J Med 19: 351353.

4. Bent JP, Kuhn FA (1994) Diagnosis of allergic fungal sinusitis. Otolaryngol Head Neck Surg 111: 580-588.

5. Schubert MS (2004) Allergic fungal sinusitis. Otolaryngol Clin North Am 37: 301-326.

6. Schubert MS, Goetz DW (1998) Evaluation and treatment of allergic fungal sinusitis. I. Demographics and diagnosis. J Allergy Clin Immunol 102: 387 394.

7. Manning SC, Holman M (1998) Further evidence for allergic pathophysiology in allergic fungal sinusitis. Laryngoscope 108: 1485-1496.

8. Coop CA, England RW (2006) Allergic fungal sinusitis presenting with proptosis and diplopia: a review of ophthalmologic complications and treatment. Allergy Asthma Proc 27: 72-76.

9. Brandwein $M$ (1993) Histopathology of sinonasal fungal disease. Otolaryngol Clin North Am 26: 949-981.

10. Corey JP, Delsupehe KG, Ferguson BJ (1995) Allergic fungal sinusitis: allergic, infectious, or both? Otolaryngol Head Neck Surg 113: 110-119.

11. Kirszrot J, Rubin PA (2007) Invasive fungal infections of the orbit. Int Ophthalmol Clin 47: 117-132.

12. Carter KD, Graham SM, Carpenter KM (1999) Ophthalmic manifestations of allergic fungal sinusitis. Am J Ophthalmol 127: 189-195.

13. Marple BF, Gibbs SR, Newcomer MT, Mabry RL (1999) Allergic fungal sinusitis-induced visual loss. Am J Rhinol 13: 191-195.

14. Elwany S, Elsaeid I, Thabet $\mathrm{H}$ (1999) Endoscopic anatomy of the sphenoid sinus. J Laryngol Otol 113: 122-126.

15. Mukherji SK, Figueroa RE, Ginsberg LE, Zeifer BA, Marple BF, et al. (1998) Allergic fungal sinusitis: CT findings. Radiology 207: 417-422.

16. Kinsella JB, Rassekh CH, Bradfield JL, Chaljub G, McNees SW, et al. (1996) Allergic fungal sinusitis with cranial base erosion. Head Neck18: 211-217.

17. Nussenbaum B, Marple BF, Schwade ND (2001) Characteristics of bony erosion in allergic fungal rhinosinusitis. Otolaryngol Head Neck Surg 124: 150-154.

18. Lund VJ, Lloyd G, Savy L, Howard D (2000) Fungal rhinosinusitis. J Laryngol Otol 114: 76-80.

19. Schubert MS (2001) A superantigen hypothesis for the pathogenesis of chronic hypertrophic rhinosinusitis, allergic fungal sinusitis, and related disorders. Annals of Allergy, Asthma \& Immunology 87:181-188.

20. Gupta AK, Bansal S, Gupta A, Mathur N (2007) Visual loss in the setting of allergic fungal sinusitis: pathophysiology and outcome. J Laryngol Otol 121: $1055-1059$

21. Carter KD, Graham SM, Carpenter KM (1999) Ophthalmic manifestations of allergic fungal sinusitis. Am J Ophthalmol 127: 189-195. 